\title{
Dermatitis herpetiformis: jejunal findings and skin response to gluten free diet
}

\author{
T REUNALA, I KOSNAI, S KARPATI, P KUITUNEN, E TÖRÖK, AND E SAVILAHTI
}

Department of Clinical Sciences, University of Tampere, Children's Hospital and Department of Dermatology, University of Helsinki, Finland; First Department of Paediatrics, Semmelweis University and Department of Dermatology, Children's Hospital Heim Pal, Budapest, Hungary

SUMMARY Fifty seven children with dermatitis herpetiformis, 18 from Finland and 39 from Hungary, were studied. Diagnostic criteria included the finding of granular $\operatorname{IgA}$ deposits in the skin of all patients. The mean age at onset of the rash was 7.2 years and favoured sites were the elbows, knees, and buttocks. Symptoms suggesting small intestinal disease were rare but in 35 $(61 \%)$ of the children subtotal villous atrophy and in $16(28 \%)$ partial villous atrophy were found on jejunal biopsy.

Eighteen children underwent a second biopsy after a mean of 21 months on a gluten free diet; villous height was found to be increased and the intraepithelial lymphocyte count decreased in all these patients. Gluten challenge caused a reversal in the two children who underwent a third biopsy. The effect of the gluten free diet on the rash was examined in Finnish children by observing the daily requirements of dapsone, a drug used to control the rash at the beginning of the diet. Eight $(67 \%)$ of the 12 children were able to stop taking dapsone after a mean of 11 months on the diet and all three patients treated with diet alone became asymptomatic after three to 6 months on the diet.

These results confirm that most children with dermatitis herpetiformis have jejunal villous atrophy, though they rarely have gastrointestinal symptoms. The central role of gluten in childhood dermatitis herpetiformis is evidenced by the fact that a gluten free diet helps the damaged jejunal mucosa to recover and controls the rash even in those children who do not have an abnormal jejunal biopsy.

Dermatitis herpetiformis in adults is characterised by a pruritic, blistering rash and is associated with coeliac disease which is mostly subclinical in these patients. ${ }^{1}$ Gluten plays an important role in adult dermatitis herpetiformis; both the enteropathy and rash respond to gluten withdrawal and relapse on gluten challenge. ${ }^{2-4}$ Dermatitis herpetiformis in children may be confused both clinically and histopathologically with other chronic, acquired bullous skin diseases but the diagnosis may be confirmed by immunofluorescence which shows granular IgA deposits in the papillary dermis. ${ }^{5}$ Previous studies using immunofluorescence in the diagnosis of dermatitis herpetiformis have been based on small numbers of children and have little or no gastroenterological data. ${ }^{5-7}$ In the present study we performed jejunal biopsies and investigated symptoms suggestive of coeliac disease in 57 children with dermatitis herpetiformis. The effect of a gluten free diet on jejunal mucosa and on the rash was investigated by performing subsequent biopsies and observing dapsone requirements in the patients on the diet.

\section{Patients}

Fifty seven children ( 33 girls and 24 boys) with dermatitis herpetiformis were examined. Eighteen were from Finland and 39 from Hungary. All children with dermatitis herpetiformis who had had jejunal biopsies performed before the age of 17 years were included in the study. The diagnosis of dermatitis herpetiformis was verified in each child by the finding of granular $\operatorname{IgA}$ deposits in the papillary dermis (Fig. 1) on direct immunofluorescence as described previously. ${ }^{8}$ The study protocol 


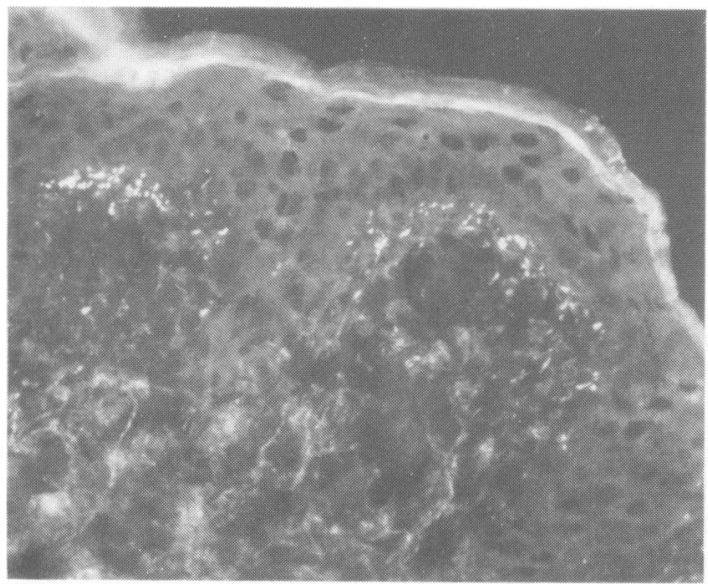

Fig. 1 Granular IgA deposits in the dermal papillae of a patient with dermatitis herpetiformis shown by direct immunofluorescence $(\times 250)$.

included asking parents about any gastrointestinal symptoms, concomitant chronic illnesses, and anaemia resistant to iron treatment. A dermatologist performed a clinical examination to give a detailed description of the rash (morphological appearance, main locations, response to dapsone (32 patients) or sulphapyridine (23 patients) and relapse on withdrawal of these drugs). The height and weight of all Finnish and 33 of 39 Hungarian children were measured at the time of jejunal biopsy.

\section{Methods}

A jejunal biopsy specimen was taken from the proximal jejunum near the ligamentum of Treiz using a Crosby-Kugler paediatric capsule. The specimens were processed and examined as described previously. ${ }^{9}$ The measurements included villous heights, crypt depths, and the counting of intraepithelial lymphocytes. Jejunal mucosa was graded as subtotal villous atrophy (villous height less than $150 \mu \mathrm{m}$ ), partial villous atrophy (villous height 150 to $250 \mu \mathrm{m}$ ), slight changes (villous height 250 to $300 \mu \mathrm{m}$ ), and normal mucosa (villous height greater than $300 \mu \mathrm{m}$ ) and the number of intraepithelial lymphocytes was expressed per 100 villous epithelial cells. Control data were obtained from jejunal specimens taken from 17 children with no gastrointestinal disorders. ${ }^{9}$

\section{Gluten free diet treatment}

Fifteen Hungarian and three Finnish children were studied to determine the effect of a gluten free diet on the jejunal mucosa. The mean age of these children was 6.9 years (range 2 to 14 years) and a second biopsy was performed after a mean of 21 months (range 12 to 74 months) on the diet. Two of the patients were challenged with gluten and a third biopsy was obtained from them after five and 12 months respectively on diets containing gluten.

Fifteen Finnish and 34 Hungarian patients were treated with a gluten free diet but because the drug requirements of the Hungarian children were not observed regularly the effect of diet on the rash was examined only in Finnish patients (mean age 12.2 years, range 3 to 16 years). Twelve of these children were treated with dapsone and diet (mean duration 37 months, range 12 to 84 months) and three with diet alone. The minimum daily dose of dapsone required to control the skin symptoms both before and during diet treatment was observed at each visit to the special outpatient clinic as described previously. $^{3}$

\section{Results}

Rash. The mean age of all the children at onset of the rash was 7.2 years; 10.9 years (range 2 to 15 years) in Finnish and 5.6 years (range 2 to 12 years) in Hungarian children. The rash in all patients was polymorphic, comprising small blisters (less than $5 \mathrm{~mm}$ diameter), macules, papules, and crusts. The main sites of the rash were the same in both the Finnish and Hungarian children-elbows (93\%), knees $(89 \%)$, and buttocks $(89 \%)$. Often the lesions were also found on the back $(70 \%)$, head $(47 \%)$ including the face $(31 \%)$, but very infrequently on the hands and feet $(5 \%)$. Pruritus was intense and in most patients some symptoms were present all the time. In $11(19 \%)$ children, however, the rash disappeared for one to three months with no treatment whatsoever and then returned. Dapsone or sulphapyridine controlled the rash well and only one patient on a normal diet was able to manage for longer periods without taking these drugs. Sulphapyridine had to be withdrawn from one patient because of hepatotoxicity and generalised dermatitis.

Gastrointestinal symptoms. At the examination, $8(16 \%)$ children presented with chronic diarrhoea and five $(10 \%)$ had a low (less than $12 \mathrm{~g} / \mathrm{dl}$ ) haemoglobin concentration. Previous gastrointestinal history was available for 48 children and included chronic diarrhoea in five $(10 \%)$, low haemoglobin concentrations in $6(13 \%)$, and failure of growth at the age of 16 months in one Hungarian patient. When the first jejunal biopsy was taken 
(mean 9.0 years, range 2 to 16 years) the mean height of the children was $-0.07 \mathrm{SD}$ for age (Fig. 2). The Hungarian children were below the mean $(-0 \cdot 3$ SD), the Finnish slightly above $(+0 \cdot 4 \mathrm{SD})$. The mean weight for the height was $-0.01 \mathrm{SD}$ and the two groups did not differ from each other in this respect. In a retrospective analysis of height and weight data from birth of 16 Finnish children no changes were observed in the growth pattern either at the time of introduction of gluten to the diet or the appearance of the rash.

Jejunal biopsy findings on a normal diet. Thirty five $(61 \%)$ of the patients had subtotal villous atrophy, $16(28 \%)$ had partial villous atrophy, three $(5 \%)$ had slight changes, and three had normal mucosa (Fig. 3). The villous height in the patients was mean (SD), 141 (89) $\mu \mathrm{m}$ and that in the controls 418 (60) $\mu \mathrm{m}-\mathrm{a}$ highly significant difference $(\mathrm{F}<0 \cdot 001)$. All patients with subtotal villous atrophy and all but one with partial villous atrophy had increased intraepithelial lymphocyte counts (greater than 35 lymphocytes $/ 100$ epithelial cells). Of the three

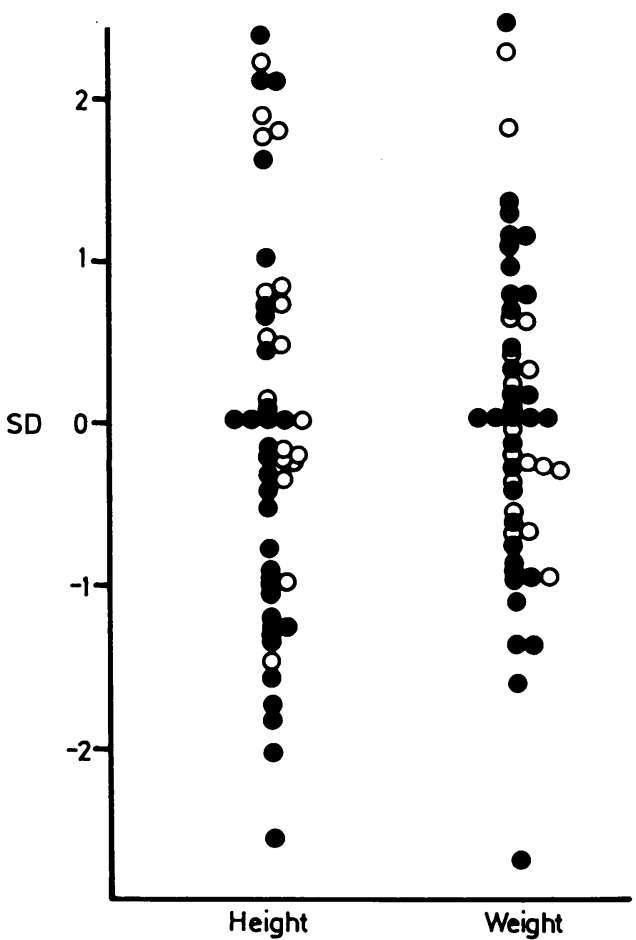

Fig. 2 Heights and weights of the 18 Finnish (open circles) and 33 Hungarian (closed circles) children with dermatitis herpetiformis when jejunal biopsy was performed. Expressed as standard deviation (SD) for age.

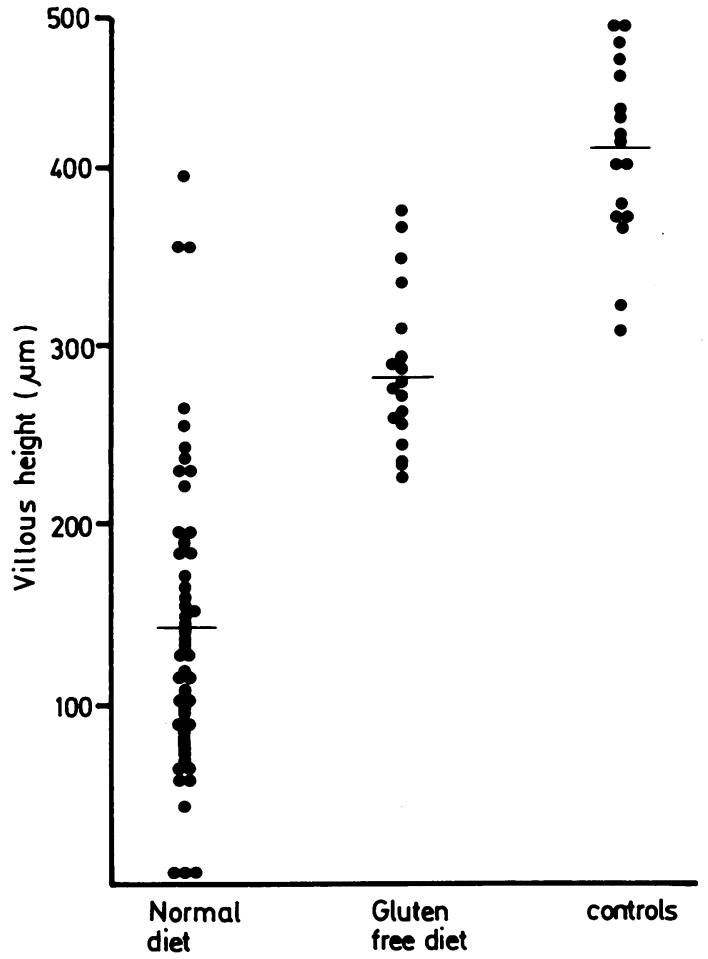

Fig. 3 Villous heights of jejunal mucosa in 57 children with dermatitis herpetiformis on a normal diet, 18 children with dermatitis herpetiformis on a gluten free diet, and 17 normal controls.

patients with slight changes, one had an increased intraepithelial lymphocyte count but the other two and the three children with normal villous height had normal intraepithelial lymphocyte counts.

Gluten free diet treatment. Eighteen patients (13 with subtotal villous atrophy and five with partial villous atrophy, villous height, mean (SD) 109 (54) $\mu \mathrm{m})$, were biopsied again while on a gluten free diet. In all patients villous height was increased and was mean (SD) 282 (47) $\mu \mathrm{m}-\mathrm{a}$ highly significant increase $(\mathrm{P}<0 \cdot 001)$. The villous height was in the normal range $(300 \mu \mathrm{m})$ in only five patients, however, and all the others had slight changes or partial villous atrophy (Fig. 3). The mean villous height: crypt depth ratio and intraepithelial lymphocyte count also shifted towards normal in each of the 18 children biopsied for a second time while on a gluten free diet (Fig. 4).

The gluten free diet caused a significant $(\mathrm{P}<0.001)$ reduction in the mean dapsone dose required to control skin symptoms in 12 children 

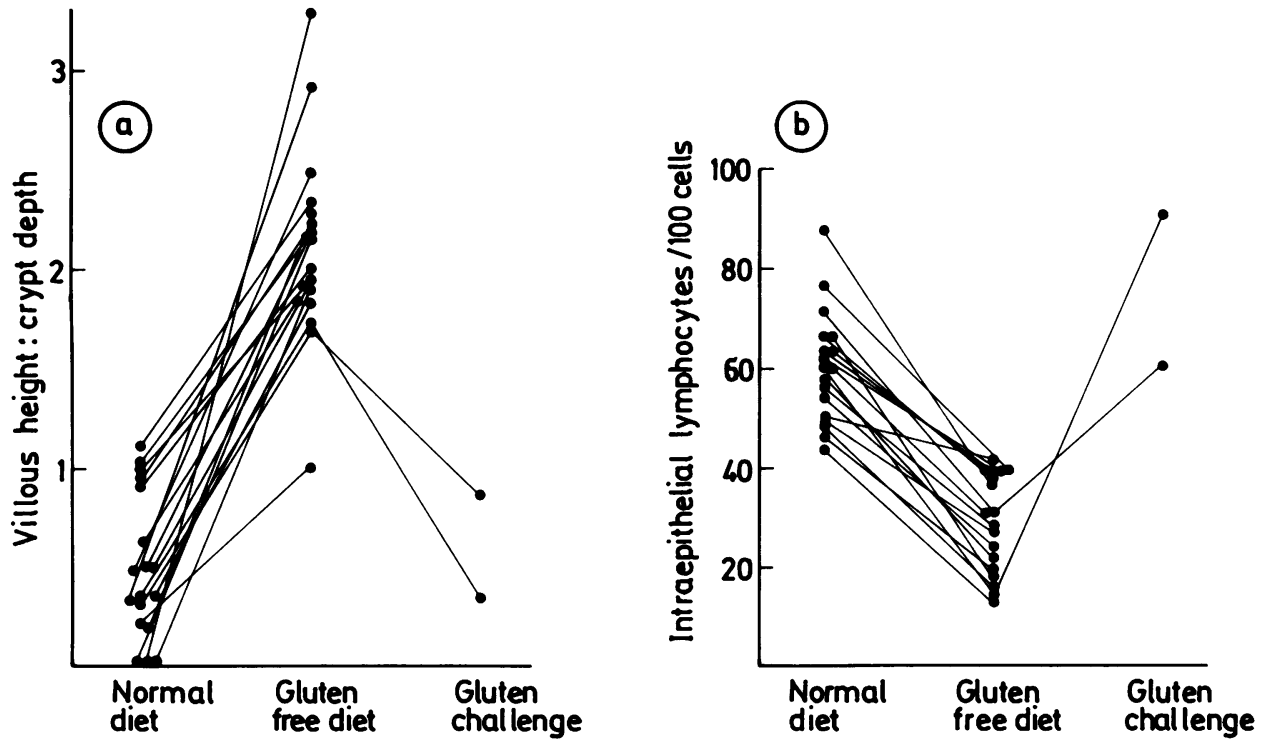

Fig. 4 Response of the jejunal mucosa to gluten free diet in 18 children with dermatitis herpetiformis. Ratio of villous height:crypt depth is increased and intraepithelial lymphocyte count in decreased in each patient on a gluten free diet.

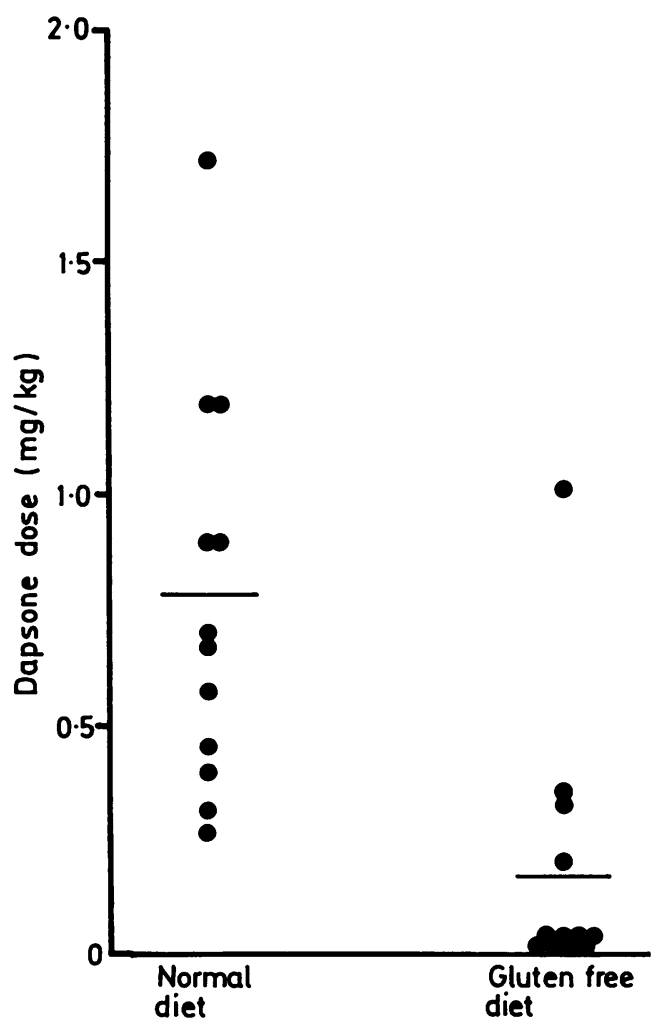

examined ( 7 with subtotal villous atrophy, two with partial villous atrophy, one with slight changes, and two with normal mucosa). In each patient the dapsone dose was reduced and in patients requiring a dose of mean (SD) $0.78(0 \cdot 12) \mathrm{mg} / \mathrm{kg}$ of dapsone to control the rash at the beginning of the diet, the dose was $0.18(0.06) \mathrm{mg} / \mathrm{kg}$ at the end of the study (Fig. 5). Eight of the 12 children were able to stop using dapsone after a mean of 11 months (range 5 to 24 months) on a gluten free diet. Two of the remaining four children were unable to follow a strict diet but even they were able to decrease the dose of dapsone. Three children (all with subtotal villous atrophy) were treated with diet alone and became asymptomatic after three, five, and 6 months on the diet.

\section{Discussion}

Marsden et $a l^{5}$ recently examined 22 children with dermatitis herpetiformis and found that granular

Fig. 5 Daily dapsone requirement $(\mathrm{mg} / \mathrm{kg})$ in 12 children with dermatitis herpetiformis on a normal diet and after the treatment with a gluten free diet (mean duration of diet 37 months). 
IgA deposits in the papillary dermis clearly differentiated between this and other acquired bullous skin diseases such as pemphigoid and benign chronic bullous dermatosis (that is linear IgA dermatosis of childhood). The present results in 57 children with dermatitis herpetiformis diagnosed on the basis of granular $\operatorname{IgA}$ deposits in the skin are in good agreement with the findings of Marsden et al ${ }^{5}$ that the rash is characterised by small intensely pruritic blisters mainly situated on the elbows, knees, and buttocks. As in adults control of the rash is dependent on continuous medication with dapsone or sulphapyridine ${ }^{10}$ and in the present study only one child on a normal diet was able to stop taking these drugs without a flare up of the rash. Spontaneous remissions occur very infrequently and the fact that childhood dermatitis herpetiformis seems to persist into adult life has been noted previously. ${ }^{5}$

Of the bullous skin diseases occurring in childhood, jejunal damage seems to occur only in dermatitis herpetiformis. ${ }^{67}$ Villous atrophy was found in each of 17 children examined by Marsden $e t$ $a l^{5}$ and in 23 of 32 children studied by Richiardi $e t$ $a l,{ }^{11}$ but in these studies no detailed data were given on the degree of villous atrophy. The present study showed subtotal villous atrophy in $61 \%$ and partial villous atrophy in $28 \%$ of the patients and the conclusion is that most children with dermatitis herpetiformis have damaged jejunal mucosaanother finding corresponding to that known to occur in adults. ${ }^{1}$

The effect of gluten withdrawal on the jejunal mucosa was examined in 18 children. Repeat biopsies while on a gluten free diet showed that the jejunal mucosa heals during the diet. Gluten free diet caused a shift towards normal mucosa in every patient as assessed by villous height, villous height: crypt depth, and intraepithelial lymphocyte counts. Villous height measurements indicated, however, that only five of the 18 patients had villous heights in the normal range and, similarly, only 10 of the 18 patients had a normal intraepithelial lymphocyte count. That the mucosal healing was not complete in every patient is in agreement with the findings in childhood coeliac disease ${ }^{12}$ and may be due to the difficulties in following a strict gluten free $\operatorname{diet}^{13}$ or to the differences in mucosal sensitivity to gluten. ${ }^{14}$

Though the jejunal disease in children with dermatitis herpetiformis is similar to that found in coeliac disease in both morphology and response to gluten free diet, the obvious difference is the infrequency of symptoms of malabsorption. Only $16 \%$ of our patients had diarrhoea at the time of examination and a previous history of diarrhoea was even more rare. Compared with childhood coeliac disease the minimal symptoms of malabsorption in our children may be due to patchy mucosal lesions, as reported in adult dermatitis herpetiformis. ${ }^{15}$ Whatever the reason, it is interesting that our children were quite old (mean age 7.2 years) when the rash began and there is now increasing awareness that older children with coeliac disease may also present with very mild gastrointestinal symptoms or even no symptoms at all. Children diagnosed as having coeliac disease when very young but failing to follow a gluten free diet in adolescence may have occasional gastrointestinal symptoms only, although they have severely damaged jejunal mucosa. ${ }^{12} 16^{17}$ Short stature with no gastrointestinal symptoms may be one sign of coeliac disease ${ }^{18}$ but in contrast to this we found no evidence of growth failure in our children with dermatitis herpetiformis.

The effect of gluten free diet on the rash in adults with dermatitis herpetiformis has been much debated but recent studies have confirmed that a strict gluten free diet followed for a sufficiently long time will control the rash, which then relapses after gluten challenge. ${ }^{3413}$ There are also incidental findings of some benefit in children with dermatitis herpetiformis. The fact that the rash is less active during the diet has been observed by some investigators. ${ }^{6710}$ The present study convincingly shows that diet treatment also controls the rash in childhood disease. Twelve children on a gluten free diet were carefully monitored and $67 \%$ of them were able to stop taking dapsone after a mean of 11 months on the diet; this percentage is similar but the time is shorter than that found in adults. ${ }^{3413}$ That treatment with diet permits control of the rash with a lower dose of dapsone or sulphapyridine, or even without these drugs, is important for minimising the risk of frequent and often dose related side effects. Both drugs can cause haemolytic anaemia, methaemoglobinaemia, leucopenia, and even fatal agranulocytosis. ${ }^{11019} 20$ Hepatotoxicity may also occur as was shown by one of our patients. An additional reason for favouring a life long gluten free diet over drugs in dermatitis herpetiformis may be the risk of developing malignancies especially gastrointestinal ones. As in adult coeliac disease ${ }^{21}$ the risk of lymphomas is very high in dermatitis herpetiformis ${ }^{22}$ but as McNeish has pointed out, ${ }^{23}$ whether or not a gluten free diet has a protective effect has not been proved. If a suitable gluten free diet is chosen, however, it can be recommended for every child with dermatitis herpetiformis irrespective of jejunal findings. As has been shown in our children and also in adults, ${ }^{3}$ the rash will respond to gluten withdrawal even if the jejunal biopsy is normal, a finding which indicates an underlying gluten sensitivity in every patient with dermatitis herpetiformis. 
In conclusion, the present study indicates that dermatitis herpetiformis occurring in children is identical to that found in adults; most of the children have jejunal villous atrophy and both the rash and the small intestinal disease respond to gluten withdrawal.

This study was supported by a grant from the Yrjö Jahnson Foundation and was performed under a Finnish-Hungarian scientific collaboration programme.

\section{References}

${ }^{1}$ Katz SI, Hall RP, Lawley TJ, Strober W. Dermatitis herpetiformis: the skin and the gut. Ann Int Med 1980;93:857-74.

${ }^{2}$ Fry L, Seah PP, Riches DJ, Hoffbrand AV. Clearance of skin lesions in dermatitis herpetiformis after gluten withdrawal. Lancet 1973;i:288-91.

${ }^{3}$ Reunala T, Blomqvist K, Tarpila S, Halme H, Kangas $K$. Gluten-free diet in dermatitis herpetiformis. I. Clinical response of skin lesions in 81 patients. Br J Dermatol 1977;97:473-80.

${ }^{4}$ Leonard J, Haffenden G, Tucker W, et al. Gluten challenge in dermatitis herpetiformis. $N$ Engl $J$ Med 1983;308:816-9.

5 Marsden RA, McKee PH, Bhogal B, Black MM, Kennedy LA. A study of benign chronic bullous dermatosis of childhood and comparison with dermatitis herpetiformis and bullous pemphigoid occurring in childhood. Clin Exp Dermatol 1980:5:159-72.

6 Thune PO, Husby G, Larsen TE, Solheim B, Ek J. Juvenile dermatitis herpetiformis and chronic acquired bullous disease in children. Clin Exp Dermatol 1978;3:279-84.

${ }^{7}$ Pehamberger H, Konrad K, Holubar K. Juvenile dermatitis herpetiformis: an immunoelectron microscopic study. $\mathrm{Br} J$ Dermatol 1979;101:271-7.

8 Reunala T. Gluten-free diet in dermatitis herpetiformis. II. Morphological and immunological findings in the skin and small intestine of 12 patients and matched controls. $\mathrm{Br} \mathrm{J}$ Dermatol 1978;98:69-78.

9 Kuitunen P, Kosnai I, Savilahti E. Morphometric study of the jejunal mucosa in various childhood enteropathies with special reference to intraepithelial lymphocytes. J Ped Gast Nutr 1982;1:525-31.
10 Marsden RA. The treatment of benign chronic bullous dermatosis of childhood, and dermatitis herpetiformis and bullous pemphigoid beginning in childhood. Clin Exp Dermatol 1982;7:653-63.

11 Richiardi P, Borelli I, Malavasi F, et al. HLA antigens in juvenile dermatitis herpetiformis. Acta Derma Venereol (Stock) 1981;61:241-4.

12 McNicholl B, Egan-Mitchell B, Stevens F, et al. Mucosal recovery in treated childhood coeliac disease (gluten-sensitive enteropathy). J Pediatr 1976;89:418-24.

${ }^{13}$ Fry L, Leonard JN, Swain F, et al. Long term follow-up of dermatitis herpetiformis with and without dietary gluten withdrawal. Br J Dermatol 1982;107:631-40.

${ }^{14}$ Falchuk ZM. Update on gluten-sensitive enteropathy. Am J Med 1979;67:1085-96.

15 Scott BB, Losowsky MS. Patchiness and duodenal-jejunal variation of the mucosal abnormality in coeliac disease and dermatitis herpetiformis. Gut 1976;17:984-92.

${ }^{16}$ McCrae WM, Eastwood MA, Martin MR, Sircus W. Neglected coeliac disease. Lancet 1975;i:187-90.

17 Stenhammar L. Transient gastro-intestinal disorders during infancy and early childhood. Acta Paediatr Scand 1981;70:383-7.

18 Groll A, Candy DCA, Preece MA, Tanner JM, Harries JT. Short stature as the primary manifestation of coeliac disease. Lancet 1980;ii:1097-9.

19 Bernstein JE, Lorincz AL. Sulfonamides and sulfones in dermatologic therapy. Int $J$ Dermatol 1981;20:81-8.

20 Sanders SW, Zone JJ, Foltz RL, Tolman KG, Rollins DE. Hemolytic anemia induced by dapsone transmitted through breast milk. Ann Int Med 1982;96:465-6.

${ }^{21}$ Swinson CM, Slavin G, Coles EC, Booth CC. Coeliac disease and malignancy. Lancet 1983;i:111-5.

22 Leonard JN, Tucker WFG, Fry JS, et al. Increased incidence of malignancy in dermatitis herpetiformis. Br Med J 1983;286: 16-8.

${ }^{23}$ McNeish AS. Coeliac disease: duration of gluten-free diet. Arch Dis Child 1980;55:110-1.

Correspondence to $\mathrm{Dr} \mathrm{T}$ Reunala, Department of Clinical Sciences, University of Tampere, 33520 Tampere 52, Finland.

Received 10 February 1984 